\title{
¿CÓMO EL VOLUNTARIADO EXTERIOR INFLUYE EN EL PÚBLICO?
}

\section{HOW DOES THE OVERSEAS VOLUNTEER INFLUENCE ON THE PUBLIC?}

\author{
Teruaki Ohnishi* \\ Shimako Funabashi**
}

\begin{abstract}
RESUMEN
El rasgo de la actividad del voluntariado ${ }^{1}$ exterior que influye en la conciencia y la actitud de las personas fue estudiado usando un modelo de sociología matemática. La actividad del voluntariado pone la conciencia del grupo participante en un estado de expectativa. Ese alcance y el rasgo del cambio de conciencia con el tiempo cambian dependiendo tanto de la fuerza de la actividad del voluntario como de la diferencia sobre la visión de valores entre este y las personas del grupo objeto. En el presente artículo se discuten de manera general el efecto de la actividad del voluntariado en el grupo de personas costarricenses y algunas implicaciones de la simulación desde la sociología matemática.
\end{abstract}

PALABRAS CLAVE: COSTA RICA * JAPÓN * COOPERACIÓN INTERNACIONAL * VOLUNTARIADO $*$ ACTITUDES $*$ SIMULACIÓN $*$ VALORES SOCIALES $*$ SOCIOLOGÍA MATEMÁTICA

\section{ABSTRACT}

The feature of volunteer's activity which influences on the consciousness and attitude of the public was studied using a model of mathematical sociology. The activity of the volunteer puts the public consciousness in a perturbed state. Its extent, together with the time-varying feature of the consciousness, change depending on both the strength of the volunteer's activity and on the difference of the view of values between the

Institute of Science and Technology for Society, Japan.

Ohnishi2015@yahoo.co.jp
1 En este artículo se utilizará el término voluntario para los profesionales enviados en misiones de voluntariado.

** Institute of Science and Technology for Society, Japan.

Shimako0930@hotmail.com 
volunteer and the society as a subject. The effect of such volunteer's activity on the Costa Ricans and some implications from the simulation are generally discussed.

KEY WORDS: COSTA RICA * JAPAN * INTERNATIONAL COOPERATION $*$ VOLUNTEERS * ATTITUDES $*$ SIMULATION $*$ SOCIAL VALUES $*$ MATHEMATICAL SOCIOLOGY

\section{INTRODUCCIÓN}

La Agencia de Cooperación Internacional de Japón (JICA) envía actualmente unos 3000 voluntarios a varios países del mundo, por ejemplo, los Voluntarios Japoneses para la Cooperación con el Extranjero (JICA, 2009a). La primera misión del voluntariado exterior de JICA es transmitir alguna técnica y conocimiento útiles para el desarrollo económico y social en aquellos países. El periodo para la actividad de cada voluntario es normalmente de dos años. En este estudio, "la actividad" se refiere a las tareas técnicas y concretas que son distintas entre uno y otro, según la especialidad profesional, y con las que cada voluntario trabaja diariamente para los grupos locales, como por ejemplo, actividades para los jóvenes, la enseñanza del idioma japonés, la terapia ocupacional y profesional para el desarrollo y la propagación de técnicas en el área rural.

Según los informes de los voluntarios, cada uno de ellos ocupa la mayoría de su tiempo diario en realizar las actividades. Sin embargo, en esos informes ha quedado de manifiesto la poca descripción sobre la evaluación de su actividad propia. Aún cuando la evaluación es realizada, es difícil obtener con alguna regularidad los resultados porque ellos explican de manera subjetiva su actividad. Aunque podemos decir que todo tiene regularidad, es apenas posible que se manifieste sobre el efecto de la actividad, ya que esta manifestación se oculta no solo en la diferencia del tipo de trabajo sino también en la manera de relacionarse con las personas, la diferencia cultural, la sensibilidad, etc. A pesar de presentarse estas dificultades en el lugar de la actividad en un estado "a tientas", la información de la regularidad sobre la previsibilidad, de cómo las personas reaccionan bajo una condición determinada y bajo un tipo de trabajo determinado, está condicionada.
En este estudio enfocamos solamente la parte sustancial de la actividad para formular un modelo, $y$ con este modelo discutiremos en general sobre ¿cómo las personas reaccionan frente a la actividad del voluntariado y especialmente frente al voluntario exterior del JICA? Hablando técnicamente, la introducción de un modelo como este nos permite evaluar no sólo predictiva sino también visual y numéricamente los resultados, $y$ puede ayudarnos a tener una comprensión colectiva. También permite discutir métodos y tácticas para la actividad. Con estos puntos de vista, este estudio discutirá la dinámica de grupo con el acercamiento de la sociología matemática e intentará evaluar la actividad cuasi-cuantitativamente.

En general, el campo y el grupo social donde el voluntariado trabaja son limitados y pequeños. El próximo punto tratará de la descripción del modelo de la dinámica que se usa en este estudio con el pequeño grupo costarricense participante. En el punto tres, el modelo se aplica a este grupo y se aprecia el proceso del cómo las personas reaccionan frente a la actividad del voluntariado antes $y$ después de que esta finaliza. Finalmente tenemos las implicaciones de esta simulación en el punto cuatro.

\section{DESCRIPCIÓN SIMPLE DEL MODELO DE DINÁMICA DE GRUPO PARA LA SOCIEDAD COSTARRICENSE}

En la estructura de la sociedad, se crea un campo de fuerza entre las personas. Dentro de este campo, las personas se influyen mutuamente y cada una cambia su conciencia. Propiamente en esta actividad, la dinámica de grupo incluye al voluntario $y$ a los miembros de este campo de fuerza. Por ejemplo, en el caso del grupo de mujeres para quienes el voluntario les transfiere una técnica y formula un modelo, 
que explica cómo reciben y reaccionan las conciencias de la personas alrededor de lo que el voluntario intenta transmitir. En este modelo, tanto los voluntarios como los individuos del grupo son partículas denominadas "agentes". Se les da seguimiento a sus movimientos en el tiempo y el espacio sociopsicológico para apreciar el estado de cambio de la posición relativa entre estas partículas. En el espacio sociopsicológico, que es abstracto, la distancia entre las partículas crea la distancia sociopsicológica, ese valor es usado como un indicador que sugiere cómo esas partículas tienen intenciones similares o diferentes mutuamente.

En este espacio se supone la acción de la fuerza sociopsicológica entre las partículas y también se supone el traslado con el tiempo de cada una de ellas, basado en el tamaño y dirección de esa fuerza. En otros términos, el agente puede modificar con el tiempo el estado mental y la conciencia sobre un asunto específico. Cuando no existe voluntariado en ese espacio, es decir, cuando no existe todavía el intento del voluntariado por transmitir una técnica en la conciencia del público objeto, las partículas correspondientes a estos últimos están en un estado de movimiento moderado con posición, dirección y velocidad seleccionadas al azar. Sin embargo, una vez que el voluntariado entra en contacto con el grupo objeto, se crea un espacio sociopsicológico entre los miembros del grupo en relación con lo que piensa el voluntario. Por consiguiente, dichos agentes crean una conciencia y una actitud sobre lo que está dentro de cada uno y las transfiere al campo de la fuerza sociopsicológica.

Este modelo, permite observar el proceso de la actividad voluntaria, captar el interés, así como el efecto de alcance del cambio de la conciencia y la actitud del grupo, por medio de la distancia que se genera entre el voluntario y cada uno de los participantes en este espacio sociopsicológico, como un elemento de juicio. Cuánto más pequeña sea la distancia cerca del cero, más cercanas están la conciencia y la actitud de los participantes hacia la del voluntario, $y$ viceversa. Cuando concuerdan las conciencias o las actitudes de las personas mutuamente, las partículas correspondientes a ellas también convergen en un punto.
Se puede indicar el método de la dinámica de opinión como una metodología para medir la diferencia de la conciencia y la opinión entre las personas participantes con la distancia relativa entre los agentes (Urbig, Lorenz y Herzberg, 2008). En la dinámica de opinión, en general, cuantificamos la conciencia o la actitud del grupo participante con un número entre $[0,1]$. También en nuestro modelo, los expresamos entre $[0,1]$ para representar la intención del voluntario y la conciencia del grupo. Si expresamos la intención del voluntariado con el número 0.25 , en este caso, el más lejano es la conciencia o la actitud de número, por ejemplo, 1 ó $2^{-1 / 2}=0.71$, que corresponden al estado de conciencia opuesto al voluntariado (véase Ohnishi (2002, 2004); Ohnishi y Viquez (2009) para el significado de aquellos números).

Desde el principio, el voluntario intenta aportar al grupo objeto una técnica y un conocimiento. Los elementos culturales del público objeto y la intención del voluntariado tienen inevitablemente sentidos diferentes en los valores, es decir, hay diferencias en la paridad (similaridad). En el caso del voluntario, quien es destinado a la misma organización donde, anteriormente, un gran número de voluntarios trabajó en la misma rama que los predecesores, las paridades de los distintos voluntarios y del público tal vez, estén igual, o muy cercanas una de la otra. Sin embargo, cuando el voluntario intenta introducir algo nuevo, su primera paridad debe ser diferente a la del público. En este caso, es necesario e importante para el voluntario, convencer al público objeto con varios métodos. En nuestro modelo, presuponemos diferencias en esta paridad.

Para observar reacciones diferentes en cada país, suponemos los coeficientes de modelo como funciones de los números de Hofstede (1997). En este estudio, examinamos la situación usando los números de Hofstede correspondientes a Costa Rica ${ }^{2}$. La unidad de tiempo

2 Teruaki Ohnishi realizó el proyecto de voluntariado, en colaboración con el Instituto de Investigación y Servicios Forestales de la Universidad Nacional, Heredia, desde enero de 2008 hasta enero de 2010. En el caso de Shimako Funabashi, lo realizó en colaboración 
es un año. Los parámetros del modelo representan el grado de la influencia del voluntariado sobre el público objeto, o la fuerza de su actividad. La norma de esta fuerza es definida como “500 ( $I \equiv 500)$ para la fuerza de la actividad en Costa Rica, con el mismo grado de influencia que una realizada en los Estados Unidos, en una campaña anti-tabaco con un presupuesto de 22,18 \$/persona/año en las relaciones públicas". En Costa Rica, para cada actividad voluntaria por ejemplo, para la educación a los jóvenes, no se ha aclarado todavía, en este momento, qué valor de la fuerza $I$ es esperada concretamente para cada caso. En la siguiente sección, observaremos la dinámica cuando el número de personas del objeto se supone en 10 . Este modelo tiene carácter estocástico y por eso el resultado que será indicado abajo no significa necesariamente que "siempre será asî" (véase Ohnishi y Viquez (2009) para los detalles del modelo).

con el Ministerio de Ambiente, Energía y Telecomunicaciones y la Cámara de Turismo de Coronado, en el Área de Conservación de la Cordillera Volcánica Central, durante los meses de marzo de 2008 a marzo de 2010.

\section{SIMULACIÓN DE LA ACTIVIDAD DEL VOLUNTARIADO EN COSTA RICA}

Todas las figuras siguientes, salvo la Figura 4, son ilustraciones superponiendo una a otra las conciencias (o las actitudes) cuantificables entre [0,1], para un grupo de diez personas. Primero, la Figura 1 representa el estado de las conciencias del grupo que cambia con el tiempo cuando el voluntariado no existe. Asumimos aquí que la conciencia de cada miembro del grupo es aleatoria en la primera etapa. Inmediatamente después de formar el grupo, cada conciencia oscila mutuamente a gran escala, pero después de los 0.3 años evoluciona moderadamente con el tiempo, y después de los dos años se forma una conciencia o una actitud unificada en todos los miembros del grupo. Esta conciencia unificada es equivalente al promedio inicial (0.5) de las conciencias del grupo.

La Figura 2 muestra la situación del movimiento de las conciencias cuando el voluntario transmite la técnica y el conocimiento y logra la misma paridad con el grupo objeto; esto sucede cuando las paridades son iguales mutuamente y la resistencia psicológica en los

FIGURA 1

LA EVOLUCIÓN DEL CAMBIO DE LAS CONCIENCIAS CON EL TIEMPO PARA DIEZ MIEMBROS DEL GRUPO OBJETO EN EL CASO DE NO-INTERVENCIÓN DEL VOLUNTARIO

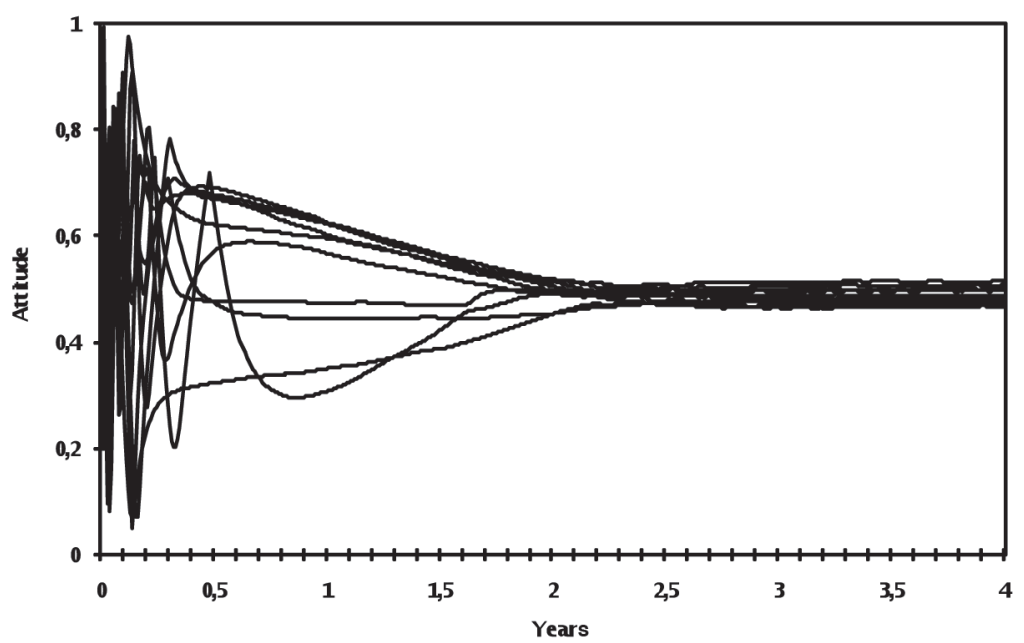


FIGURA 2

EL CAMBIO DE CONCIENCIAS DE DIEZ MIEMBROS EN EL CASO DE LAS PARIDADES IDÉNTICAS

ENTRE EL VOLUNTARIO Y EL GRUPO OBJETO. EL PERÍODO DE LA ACTIVIDAD DEL VOLUNTARIO

CORRESPONDE SOLAMENTE A LOS PRIMEROS DOS AÑOS. (A) $I=50$, (B) $I=500$, Y (C) $I=5000$, DONDE $I$ ES

EL GRADO DE ACTIVIDAD.
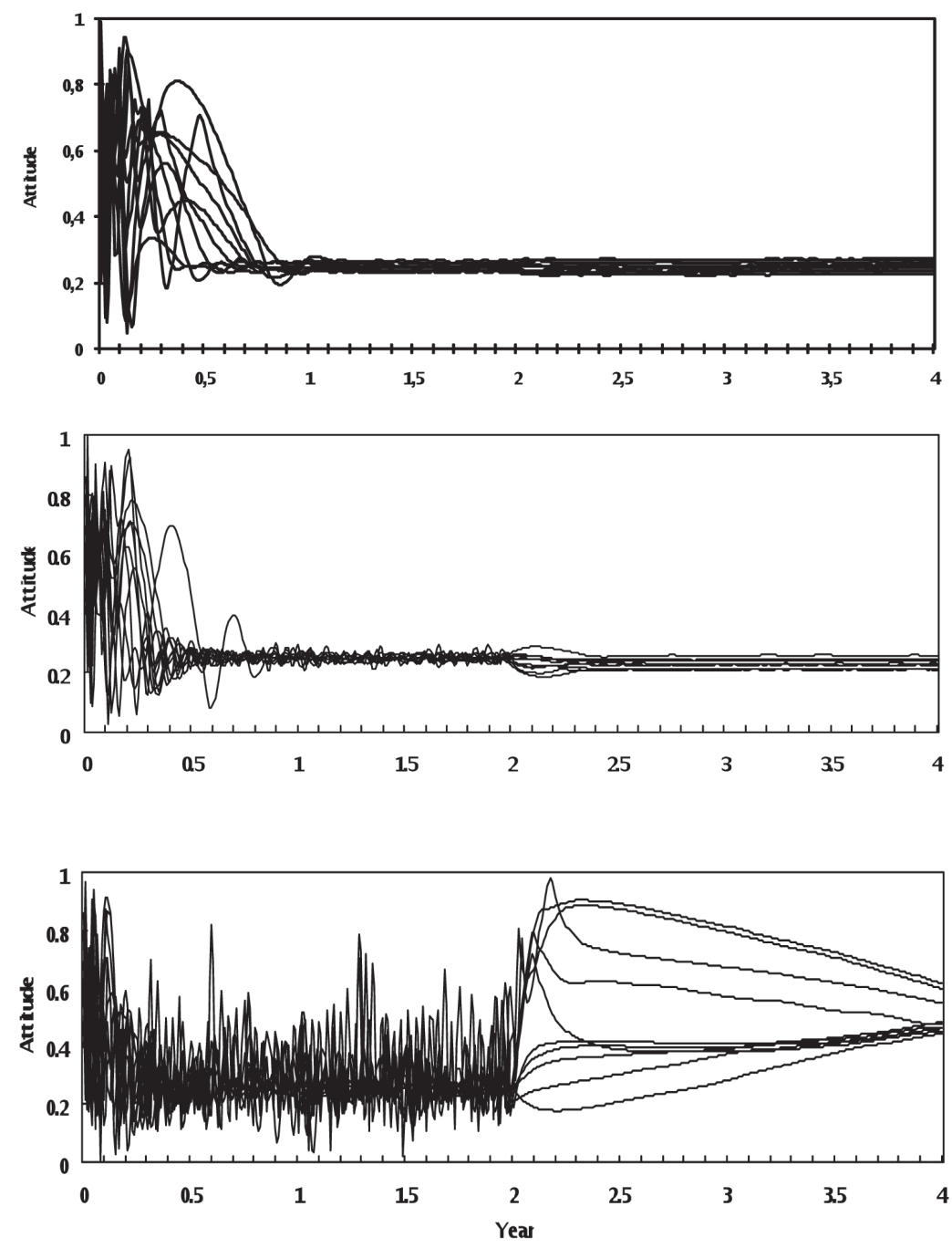

miembros del grupo es pequeña. En este caso, para la ejecución de su trabajo, el voluntario está obligado solamente a orientar al público en la dirección de sus propias intenciones. Hay un período de resistencia por parte del grupo objeto, este gradualmente entenderá la intención del voluntario (0.25), interiorizando su propósito siempre y cuando la actividad no se ejecute con fuerza excesiva. Si es poca la resistencia a la actividad I (Fig.2(a)), se da un cambio progresivo en la conciencia y en la actitud; por consiguiente podemos decir que la técnica y el conocimiento son transmitidos inmejorablemente a los miembros del grupo. Cuando la cantidad $I$ es pequeña, podría decirse que el voluntario logró entablar vínculos entre él y el grupo objeto. Por lo tanto, el estado de la incorporación de las conciencias y las actitudes de los miembros persisten con el paso del tiempo, aunque el voluntario se aparte del grupo objeto. 
Cuanto más intensamente crezca la actividad del voluntariado, más fuerte será la disposición de las personas participantes que son cautivadas por esa actividad, oscilando sociopsicológicamente alrededor de la intención del voluntario. En el caso de $I=500$, luego de que el voluntario se aparta del grupo, los participantes pierden el poder de agruparse durante unos meses (Fig.2 (b)). Sin embargo, después de algún tiempo sus consciencias se unen de nuevo. Si la actividad transcurre durante dos años, este valor condensado de nuevo, que es el valor medio (0.23) de las conciencias de los miembros en ese momento, es aproximadamente igual al valor (0.25) existente durante el período de trabajo voluntario. Este estado de separación psicológica y el valor de las conciencias condensado de nuevo están más propensos a la influencia del retorno del voluntariado, con el incremento del período de actividad. Esto corresponde a que la intención del voluntario es aceptada por el grupo objeto.

Por otro lado, cuando la actividad demanda mucha exigencia, el grupo participante siempre es presionado psicológicamente. Una actividad así como esta, que obra a su antojo sin prestar la debida atención al tiempo de respuesta del grupo lleva consigo una notable dispersión psicológica entre los participantes después de la salida del voluntario. En este modelo, asumimos que la acción mutua (como las reuniones, varios intercambios de información, y el trabajo cooperativo) sobre el proyecto en cuestión, permanezca en el grupo aún después de la salida del voluntario, así como durante el período de su trabajo. Al no existir acción mutua de este tipo, la psicología del grupo subsiste individualmente y la cohesión no se realiza de nuevo después de su salida.

La Figura 3 indica la variación de la conciencia (o la actitud) del grupo en el caso en que la intención de voluntario difiere del valor del grupo objeto, es decir, cuando los participantes muestran una paridad completamente diferente a la del voluntario. La intención del voluntariado está en 0.25 , como la figura 2 , y los valores 1.0 y 0.71 que están lejos de este 0.25 , indican el estado de la conciencia de las personas que rechazan al voluntario, mostrándole antipatía. Una vez que los participantes cambian su actitud por la persuasión del voluntario, la conciencia de los primeros es atraída por la del voluntario en 0.25 (es decir, se crea un lugar geométrico que traslada con el tiempo rápidamente los valores de 1.0 ó 0.71 muy cercanos al 0.25), y luego entra en estado fluctuante sobre este valor de 0.25. En el caso mostrado en la Fig.3(c) correspondiente a la actividad voluntaria intensa $y$ muy emprendedora $\left(I=5 \times 10^{3}\right)$, el comportamiento del grupo objeto después de la unificación de su paridad con la del voluntariado (aproximadamente medio año) entra en un estado parecido a la del grupo que tiene la paridad igual a la del voluntariado al comienzo de su actividad. En otras palabras, en este caso, aunque la conciencia del grupo esté de acuerdo con la del voluntario por su actividad durante medio año, estos no se acercan siempre, sino que su conciencia y su actividad es fluctuante entre estable e inestable alrededor de la intención del voluntario. Al cabo de dos años, con la salida del voluntario, la conciencia (el interés) de las personas involucradas se separa rápidamente.

Es evidente que cuánto menor sea el grado de actividad del voluntario, más difícil será para él captar la atención del grupo objeto. En las intensidades de $I=10^{3}$ (Fig.3(b)) y $5 \times 10^{2}$ (Fig.3(a)), los porcentajes del grupo, que tienen la actitud conforme a la intención del voluntario son 80 y 30 porciento respectivamente. Con una actividad-menos intensa, como $I<10^{2}$, aunque puede causar conmoción y fluctuación en la psicología del grupo, no provoca el cambio esencial en la actitud y la forma de pensar. En este caso, el voluntario es incapaz de persuadir a una persona incluso dentro del plazo de los dos años.

Con la salida del voluntario al cabo de dos años, su influencia trasciende hasta las personas que no han cambiado su actitud, es decir, él es vínculo que une a los miembros hasta su salida dentro de cada grupo. Tanto los que aprueban como los que no al voluntariado, la influencia se vuelve más débil, haciendo que la conciencia de los miembros en cada grupo alcance un estado de separación (como la situación de dos a tres años en las Figs.3 (a) y 
FIGURA 3

LO MISMO QUE LA FIG.2, PERO EN EL CASO DE LA INDIFERENCIA DE PARIDADES ENTRE AMBOS. (A) $I=500$, (B) $I=1000$, Y (C) $I=5000$.
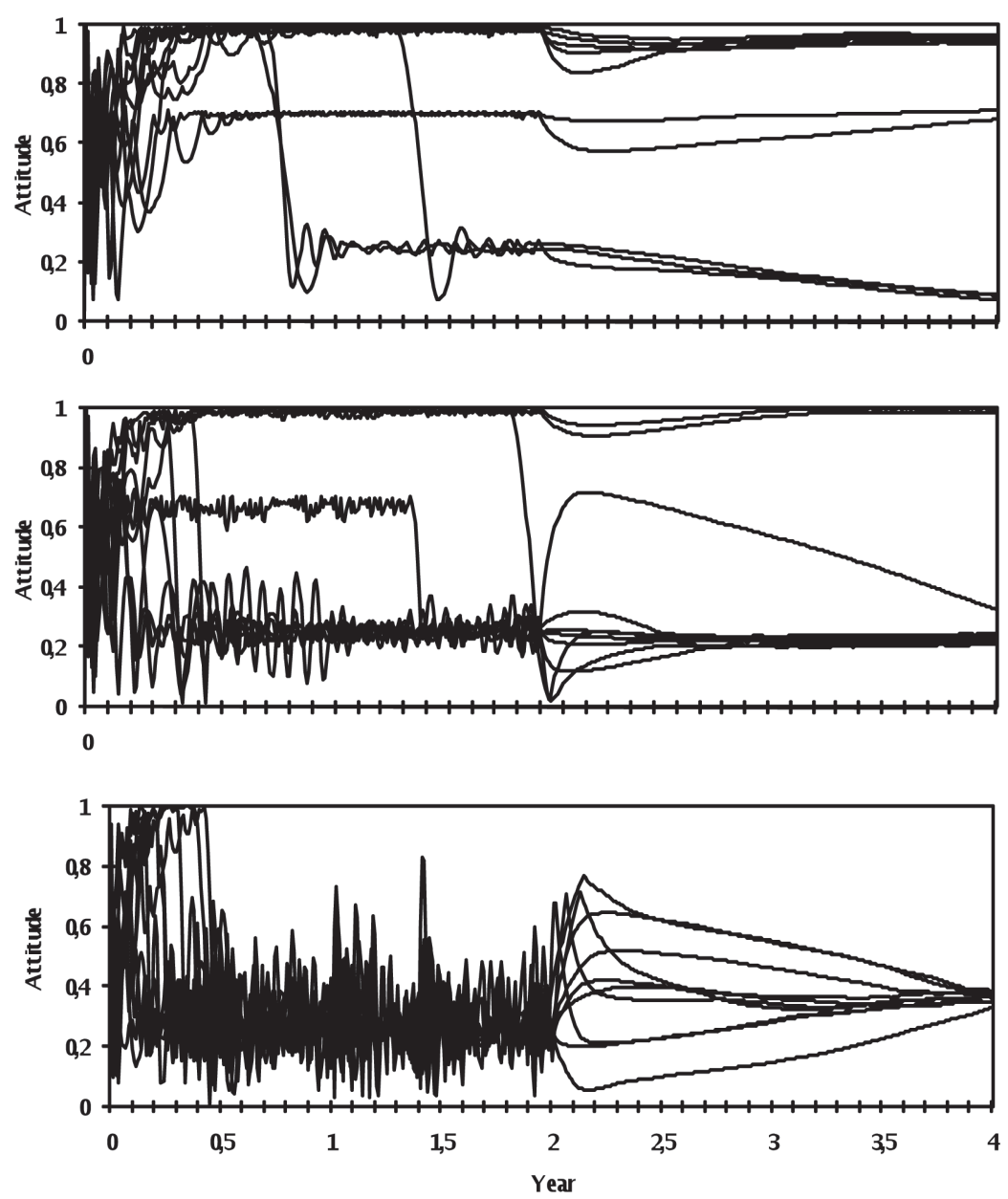

(b)). Sin embargo, igualmente en el caso de la Fig.2, siempre que la interacción persista dentro del grupo, el estado de la agregación psicológica es alcanzado de nuevo por estos. En general, en el caso de una actividad menor, la fluctuación de la conciencia de los participantes que aceptan al voluntario es insignificante hasta que se produzca un cambio de actitud, con tendencia a mantener un estado relativamente estable (la situación cerca de 0.25 de uno a dos años en la Fig.3(a)). Por eso, la parte del grupo desinteresada no es necesariamente muy influenciada por la salida del voluntario y su estado de aglomeración psicológica no cambia aunque su conciencia lo haga gradualmente con el tiempo, en comparación con el período en el que el voluntariado estaba presente (tres curvas mostradas bajo la región a partir de dos años en la Fig.3 (a)). A pesar de que este escenario tiene condiciones de actividad débil y estas son más favorables, puede aparecer un cambio muy pequeño en la conciencia y la actitud de esas personas. En este caso, se llega solamente al estado en que muchas de ellas mantienen su manera de pensar $y$ su actitud tradicional.

Asumimos el porcentaje del grupo objeto, que cambió su manera de pensar y su 
actitud, como la eficiencia de la actividad $\varepsilon$ del voluntario. La eficiencia de su actividad tiene un límite superior dependiendo de la intensidad de la actividad, en el caso del trabajo por un periodo de dos años. Además en este caso, hay un valor de umbral sobre la intensidad de la actividad por la cual se puede obtener un efecto significativo durante el periodo de actividad. Este aspecto es ilustrado en las curvas de nivel en la Figura 4.

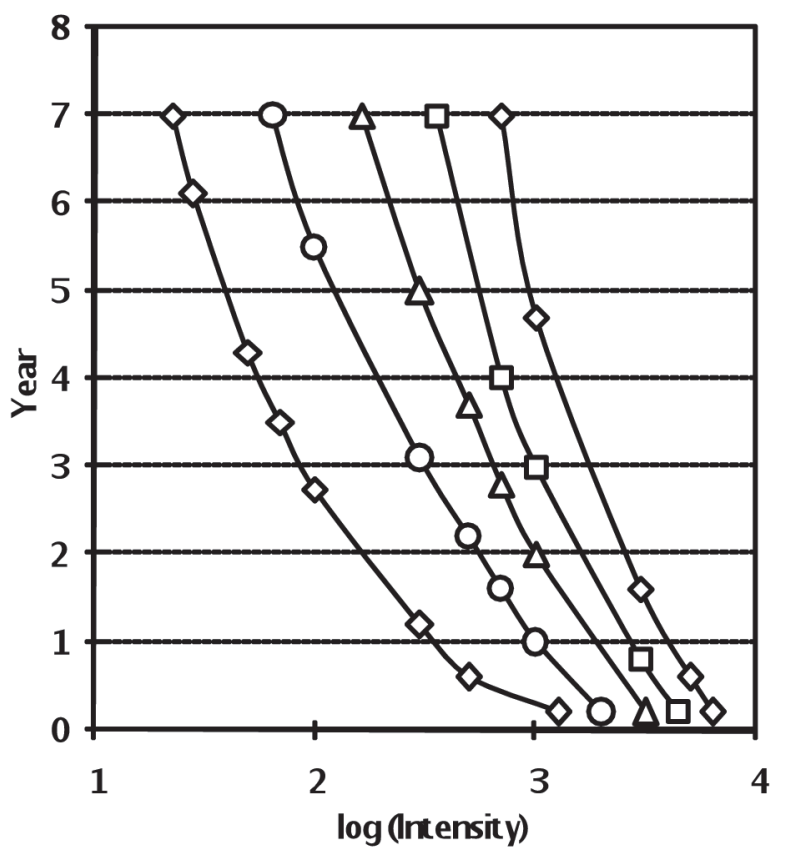

\section{IMPLICACIÓN}

El resultado del punto anterior es propio para el caso costarricense, $y$ por eso es determinado dependiendo del carácter nacional. Según Hofstede (1997), el valor del Individualism (individualismo) es generalmente muy bajo en todos los países de Latinoamérica. Costa Rica no es la excepción. La característica del bajo Individualism es el fuerte colectivismo que corresponde a: tener una tendencia marcada sobre la dependencia de la propia identidad al grupo que se pertenece. Esto corresponde a la posesión de una fuerte norma social y corresponde también a la existencia cotidiana de una fuerte presión social. Por eso, la tendencia para el individuo, del cambio de opinión o actitud según la persuación por el voluntariado, es débil. En una sociedad así, el voluntariado necesita ganar la confianza de todos los miembros del grupo, para lograr el cambio en sus conciencias $y$ actitudes.

Debido a la presión social, se supone que el costarricense es muy conservador frente a la alteración de la visión de valores y de la visión de mundo (Biesanz, Biesanz y Biesanz, 1999). Por eso, no es fácil alterar conceptos abstractos como la actitud mental y la manera de ser; no es exagerado decir que la acción individual está basada en los beneficios esperados concretamente, más que en esos conceptos. En estas situaciones, la técnica transmitida por el voluntariado puede permanecer hasta cierto punto cuando trae algún beneficio. El voluntario, quien trabaja en las comunidades rurales, se encuentra frecuentemente con irracionalidades 
entre los participantes costarricenses, $y$ él ha intentado guiar al grupo objeto en la dirección que él considera racional dentro del concepto. Pero en ese caso, es difícil lograr un buen resultado de esta acción en Costa Rica, y el voluntario, con frecuencia siente impotencia, como fue escrito en el informe de un voluntario japonés enviado a Costa Rica como "la persuasión para el individuo costarricense es insignificante".

Generalmente, el accionar del voluntariado tiene como objetivo introducir métodos actuales desconocidos, es decir, prácticas distintas a las existentes, para el grupo objeto en esa área específica. En este caso, como se mencionó en el capítulo anterior, podemos decir lo siguiente:

1) Cuando el voluntariado trabaja intensamente, es posible alcanzar, relativamente sin dificultad, un cambio en la manera de pensar y en la conducta. Sin embargo, la psicología y la actitud de las personas oscilan notablemente después de este cambio.

2) Cuando el trabajo del voluntariado es moderado, el efecto de persuasión en el público es insignificante. No obstante, la disposición psicológica de los participantes va cambiando y su paridad se acerca, con el tiempo, a la del voluntario al presentar correspondencia $y$ estabilidad en su actitud. En este caso, no se presenta fluctuación excesiva psicológicamente en el grupo, aún ante la salida del voluntario. Por otro lado, la unidad psicológica retorna al estado original después de un tiempo finito, el que se explica porque los participantes estuvieron separados debido a la pérdida del poder de convocatoria para el ejercicio de la psicología colectiva.

Para obtener finalmente, un cambio estable de la conciencia y la actitud de los grupos, no solo debe considerarse cualidades de uno de los dos casos antes mencionados, sino también la combinación de ambos. Es decir, el voluntariado debe-lograr un cambio en el pensamiento $y$ la actitud de todas las personas, primeramente participando con actividad intensa, y luego, debe con acción débil atraerlos de manera estable, alrededor del objetivo del voluntariado. Los ejemplos para esto se muestran en la Figura 5; mientras que la Fig.5 (a) representa el caso en que un primer voluntario realizó su actividad durante un año con la intensidad de $5 \times 10^{3}$, posteriormente, después de un año de su salida, un segundo voluntario realiza una actividad con intensidad débil de $5 \times 10^{2}$ durante dos años, la Fig.5 (b) representa el estado del cambio de conciencia del público en la actividad de $5 \times 10^{3}$ en el primer año y $5 \times 10^{2}$ en el segundo año. Es evidente en estas figuras que el método de cambio en la intensidad de la actividad con el tiempo puede ser una manera más efectiva para persuadir al grupo objeto. Asimismo, cambiar el carácter $y$ el contenido de la actividad, con el tiempo puede ser también otra manera efectiva para la persuasión.

Finalmente, siguiendo nuestro modelo, queremos interpretar la trayectoria de un voluntario que trabajó activamente en Costa Rica (Ishizu, 2006; JICA,2009b).

El Sr. Ishizu estuvo destinado a una oficina local del Ministerio de Agricultura y Ganadería en diciembre de 2004 como voluntario para la enseñanza estandarizada de una manera de cultivo de las verduras. Él fue el tercer voluntario del JICA destinado a esa oficina, por eso las personas ya estaban familiarizadas con los voluntarios japoneses. La intención de su estadía en Costa Rica era popularizar el cultivo orgánico de las verduras y él no tuvo dificultad para recomendarles esa manera de cultivo ya que muchas mujeres deseaban adoptarla. Esta aceptación se debió también al interés que mostraron las mujeres pues ellas ya tenían algún conocimiento de esta práctica que les permitía cultivar fácilmente las verduras sin ensuciarse las manos. En este caso podemos interpretar que Ishizu y el grupo de mujeres mantenían la misma paridad desde el comienzo de su actividad, por eso su técnica y su conocimiento fue recibido por este grupo sin dificultad.

Después de medio año de la terminación de su actividad, Ishizu fue enviado de nuevo a la oficina que tenía asignada anteriormente como 
FIGURA 5

EL CAMBIO DE CONCIENCIAS PARA LOS MIEMBROS DE UN GRUPO EN EL CASO EN QUE CUANDO LA INTENSIDAD DE LA ACTIVIDAD CAMBIA CON EL TIEMPO CONTRA FRENTE AL GRUPO CON LA PARIDAD DIFERENTE DE LA DEL VOLUNTARIO. (A) EL CASO DE LA INTERVENCIÓN DEL VOLUNTARIO CON $I=5000$ EN EL PRIMER AÑO, LA NO-INTERVENCIÓN DURANTE EL SIGUIENTE AÑO, LUEGO LA INTERVENCIÓN CON $I=500$ DURANTE LOS SIGUIENTES DOS AÑOS, Y ADEMÁS LA NO-INTERVENCIÓN DE NUEVO. (B) EL CASO DE LA INTERVENCIÓN CON $I=5000$ EN EL PRIMER AÑO, CON $I=500$ EN EL SIGUIENTE AÑO, Y LUEGO LA NO-INTERVENCIÓN.
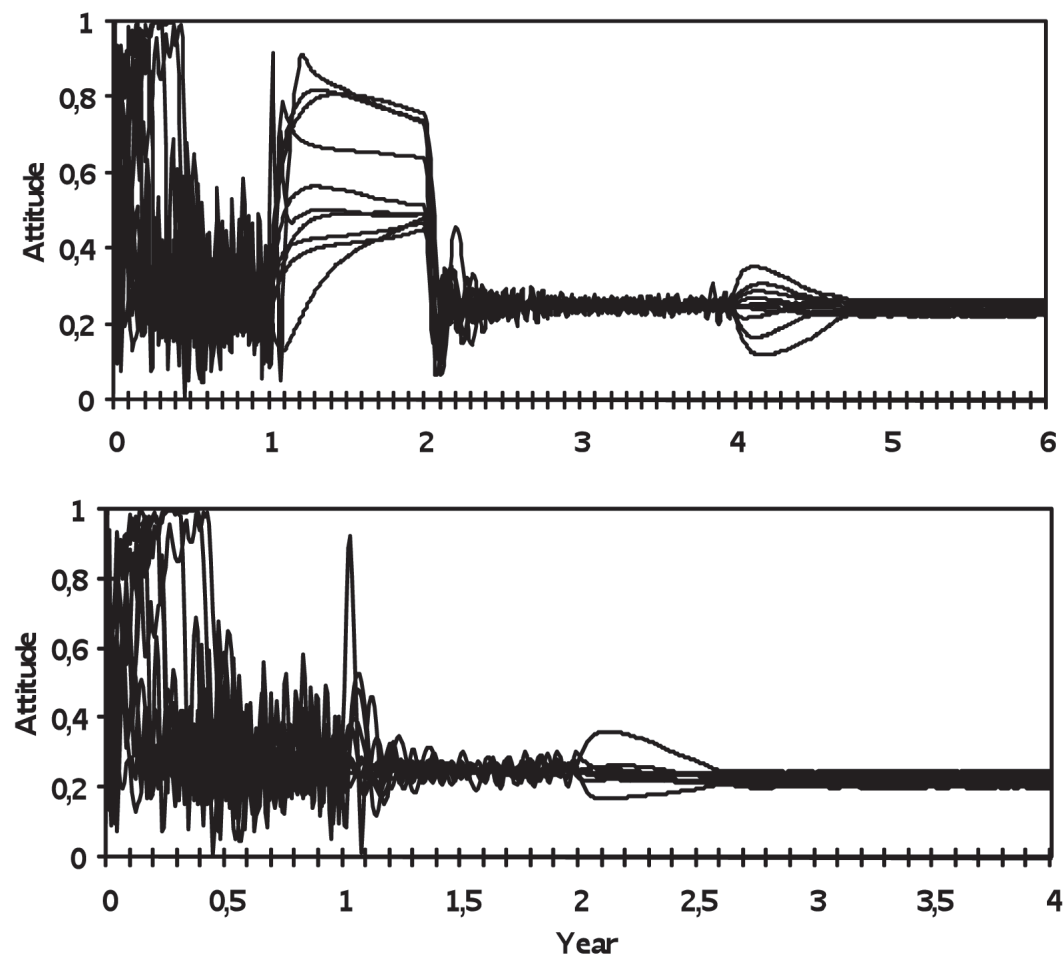

un voluntario de corta duración, y además otro voluntario con la misma especialidad profesional que la suya, fue enviado como su sucesor. Aún, después de su salida en diciembre de 2006, el grupo de mujeres continuó el cultivo de verduras por ese método. Esta situación corresponde a los casos de las Figs. 2(a) o (b). Según nuestro modelo, la técnica de ese método de cultivo será mantenida probablemente aunque Ishizu esté ausente, siempre que persista la comunicación dentro del grupo como en los inicios. Por eso, podemos decir que su actividad de nuevo en la oficina y también la introducción de su sucesor apenas fueron necesarias. En este caso, aunque no necesariamente debe ser fuerte, la existencia de un tipo de atractor [aquí definido como algo que en lugar del voluntario llama más la atención del público $y$ atrae su conciencia, por ejemplo, el boletín en la oficina y su difusión entre el personal] es posible que sea más efectivo para la continuación y el mantenimiento de la conciencia o la actitud del grupo (Ohnishi y Viquez, 2009). De esta manera, para la continuación y el mantenimiento más efectivo del cultivo orgánico, Ishizu debió crear jefes quienes se convirtieron en atractores dentro del grupo antes de su salida en diciembre de 2006. En caso de hacer esto, no sería necesario su envío de corta duración y tampoco su sucesor. Es decir, en el caso de Ishizu, fue necesario el cambio oportuno del carácter y el contenido de su actividad con el tiempo.

Desde este punto de vista, en el caso del envío sucesivo de muchos voluntarios a la misma organización, cada carácter y cada 
contenido de sus actividades deben de diferenciarse necesariamente, aunque estos tengan la misma especialidad profesional. Es muy importante, para aumentar el efecto de la actividad voluntaria, requerir fuertemente que todo los voluntarios tengan la comprensión correcta sobre en qué lugar del proceso de persuasión al grupo se sitúa su rol como voluntario.

El foco de este estudio está limitado sólo a la actividad realizada por los voluntarios provenientes de Japón. Sin embargo, decimos sobre la comprensión cualitativa, que la discusión en este estudio seguramente puede corresponder a todos los voluntarios exteriores en cualquier país, y a todos los países donde trabajan los voluntarios exteriores.

\section{REFERENCIAS}

Biesanz, M.H.; Biesanz, R. y Biesanz, K.Z. The Ticos: Culture and Social Change in Costa Rica. Boulder, Colorado: Lynne Rienner Pub, 1999.

Hofstede, G. Culture and Organizations: Software of the Mind. New York: McGraw Hill, 1997.

Ishizu, T. Informe final de la actividad por Voluntarios Japoneses para la Cooperación con el Extranjero por T.Ishizu en Costa Rica (en japonés). Tokyo: JICA, 2006.
JICA. "JICA". En: <http://www.jica.go.jp/english/ index.html>. 2009a.

JICA. "JICA Voluntario Antes/Despúes: Sr. Tomohisa Ishizu estuvo impaciente en Costa Rica, por eso se decidió hacer la agricultura orgánica en Japón” (en japonés). Cross Road 3. 2009b: 32-33.

Ohnishi, T. "A multi-particle model applicable to social issues-time evolution of Japanese public opinion on nuclear energy". Annals of Nuclear Energy 29. 2002: 1747-1764.

Ohnishi, T. "The Effect of Opinion Leader on Nuclear Public Opinion - Its Visualization on a Socio-psychological Plane". Journal of Nuclear Science and Technology 41. 2004: 152-162.

Ohnishi, T. y Viquez, A. To What Extent does Persuadability of the Public Depend on their Culture?. En: <http://sts.or.jp/ EngFiles/TOAV.pdf >. 2009 .

Urbig, D.; Lorenz, J. y Herzberg, H. "Opinion Dynamics : The Effect of the Number of Peers Met at Once". Journal of Artificial Societies and Social Simulation 11. 2008. En: <http://jasss. soc.surrey.ac.uk/11/2/4.html $>$ y las referencias allí. 
